\title{
Dynamic optimization of the LNG value chain
}

\author{
Bjarne A. Foss ${ }^{\mathrm{a}}$ and Ivar J. Halvorsen ${ }^{\mathrm{b}}$ \\ ${ }^{a} N T N U$, Department of Engineering Cybernetics N-7491 Trondheim, Norway \\ ${ }^{b}$ SINTEF ICT, Applied Cybernetics, N-7465 Trondheim, Norway
}

\begin{abstract}
In operation of a large LNG processing plant there will be uncertainties related to planning and realization of an optimal operation strategy. Results show the importance of including the whole production chain in the optimization. For a test scenario where we look at the event of delayed ship arrival, this gives lower losses than a normal approach where the upstream part of the system and the LNG process plant are optimized individually. The system is modeled by simple models of each main component starting at the wells and near-well region and ending with the export tanks for LNG, LPG and condensate. These proxy models can be regarded as first order approximations of the real system. The individual models are nonlinear, some are static and others are dynamic models. The models have been coarsely validated using a highfidelity simulator of the value chain at the Snøhvit LNG plant. Use of these quite simple models combined with model-based optimization offers and interesting and feasible approach to optimize production in case of various events that require readjustment of the production planning.
\end{abstract}

Keywords: Production chain, LNG, simplified models, optimization

\section{Introduction}

Optimal operation of large LNG processing plants may require a holistic view of the value chain. This is important if the LNG plant is tightly connected to an upstream production system and/or a downstream consumer. Present industrial practice typically takes a silo approach in the sense that one part of the supply chain is treated quite separate from other parts. This is pronounced in the upstream area where applications for optimally allocating well production include well models as well as models for some pipeline collection system. The downstream boundary condition is typically a constant pressure in a $1^{\text {st }}$ stage separator. Similarly model-based optimizers for the separation plant do not include models of the upstream part. Instead information from the upstream part is passed as an exogenous variable including flow rates, pressure and composition to the downstream optimizer. This implies that the inlet separator functions as a "dividing wall" between the two optimizers even though the two subsystems might be tightly connected. An example of the latter arises if part of the gas output from the separation plant is fed back into the upstream system through gas-lift wells or gas injectors. There are many reasons for this silo-like situation. Different parts of the supply chain recruit people with different backgrounds and they use different tools for optimization. This limits integration even in situations where integration has an obvious potential. 
We study how model-based optimization can be used to find an optimal operation strategy in the presence of unexpected operational events focusing on delayed arrival times for ships exporting stored products. In particular the potential of combining upstream production system with the downstream process plant into one optimization problem and the use of simple models is explored. The system we study is a semirealistic model of the production chain of StatoilHydro's Snøhvit plant in Hammerfest, close to Norway's North Cape. The wells are situated about $120 \mathrm{~km}$ from shore, and gas condensate is transported to the processing plant in Hammerfest through a multiphase pipeline driven by the well pressure only. In addition to the central liquifaction unit, the plant consists of gas pretreatment units, a gas-fired power plant, a $\mathrm{CO}_{2}$ separation unit and storage tanks. LNG and condensate products are shipped to customers worldwide. It may be noted that $\mathrm{CO}_{2}$ is sequestered in an aquifer. A stylistic view of the plant is shown in Fig.1.

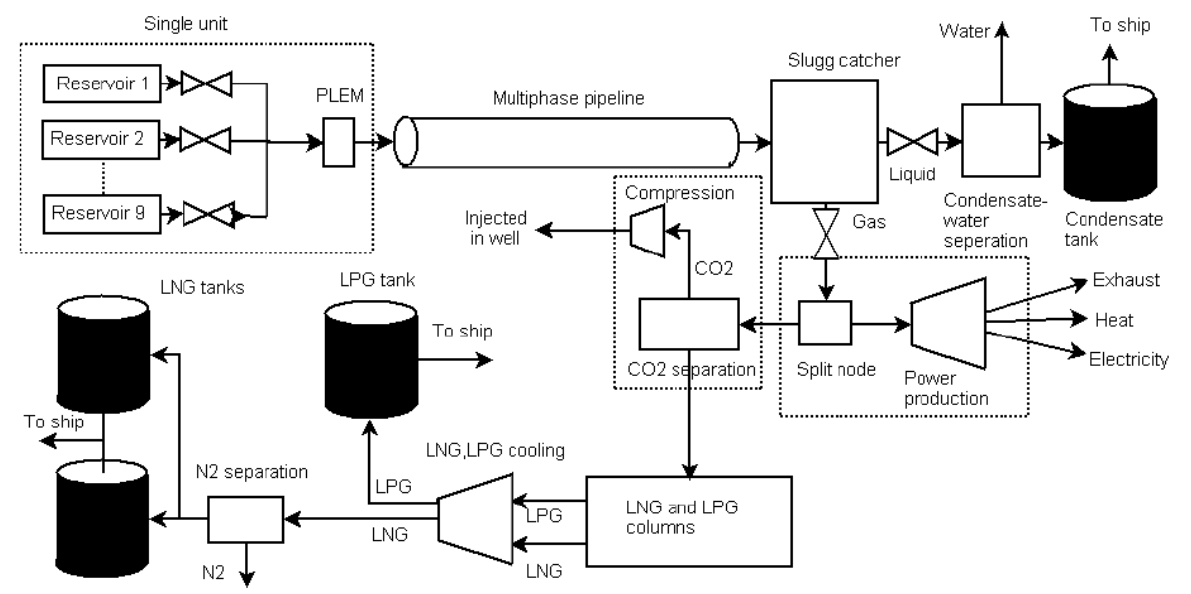

Figure 1: A stylistic view of the Snøhvit production chain (Dahl, 2007)

The paper first places the proposed method into context by relating it into a wellestablished process control hierarchy. Subsequently the modeling approach and the optimization approach are presented. Thereafter a plausible test scenario is described together with simulated results. The paper ends with a discussion and some conclusions. Page constraint limits the depth of the presentation. References are therefore given for readers who seek more detailed information on the method and results.

\section{A process control hierarchy}

The Snøhvit reservoir fluid is extracted subsea, transported in multiphase pipelines, refined and liquefied, stored, shipped, regasified, stored, transported through some gas pipeline network and before it finally can be used for heating or as raw material. Not only is the chain long and the transport methods varied, there are also many important decisions to be made by partners regarding and related to marked, pricing, stocking, production level and production security. Some supply chains are directly connected 
(like natural gas used in fertilizer production), others are more decoupled (like the consumption of electricity produced at a gas power plant). Hence, not "all” parts are equally important to include in a supply chain. There are different methods and applications for managing supply chains. We use the well established process control hierarchy (see e.g. Backx, Bosgra and Marquardt, 2000) as a starting point keeping in mind that the process plant is in turn only a part of a larger supply chain, see also Fig.2. The levels are:

- Regulatory control: The low level control of processes are essential for keeping the plant stable and well functioning. This level usually consists of conventional PIDcontrollers.

- Supervisory control: This layer usually handles units and may be based on manual adjustment of the regulatory layer controller setpoints or model predictive control (MPC).

- Local optimization: Adjust targets to maintain close to optimal operation under changing conditions. This layer is often based on real time optimization (RTO) with a model fitted to real time data.

- Site-wide optimization: For large plants, there will usually be a plant wide RTO level, coordinating sections and units.

- Scheduling and planning Planning is closely connected to the whole supply chain. The planning level can again be subdivided into long-term (e.g. investment decisions), mid-term (e.g. season planning, sales contracts, maintenance planning), and short-term plans. The latter includes setting targets for the site-wide optimization, handling discrete operations like ship arrivals and loading and re-planning in case of unexpected events.

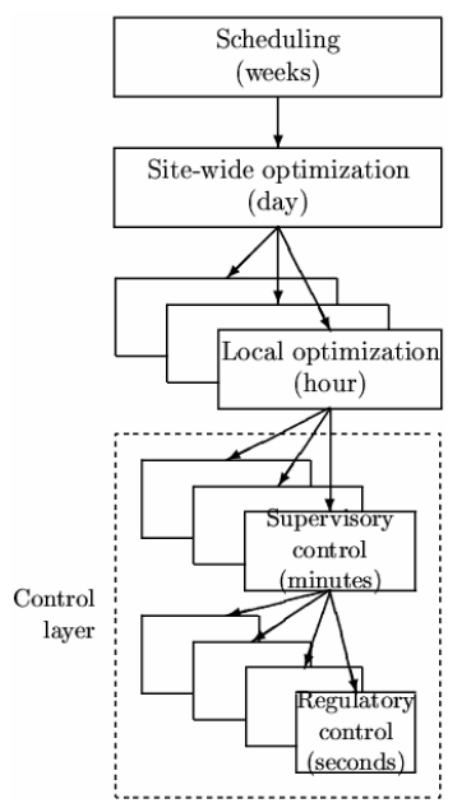

Figure 2. Control hierarchy

The focus in this paper is the connection between short-term planning and site-wide optimization.

\section{LNG plant modeling approach}

The choice of model complexity is critical for the success of a model-based optimization. On the one hand the model must be accurate in the sense that optimization provides reasonable results. On the other hand the model must be simple, or at least 
computationally efficient, such that the optimization problem can be solved within the time constraints at hand.

\subsection{Model structure}

Our approach is to model the system using a modular structure with simple models of each main component starting at the wells and near-well region, and ending with the export tanks for LNG, LPG and condensate. More precisely the modules include: wells, pipeline, slug catcher, condensate column, condensate tank, $\mathrm{CO}_{2}$-separator, LNG and LPG column, gas turbine, LNG and LPG cooling compressors, $\mathrm{N}_{2}$ separator, LNG tank, and LPG tank, see also Fig.1. Some auxiliary systems like the floe assurance system is not included in the model.

The complete model can be viewed as a first order approximation of the real system. The individual models are nonlinear, some are static and others are dynamic models. The models have been coarsely validated using a high-fidelity simulator of the value chain at the Snøhvit plant (Dahl, 2007).

The simulation model was created to solve some specific optimization scenarios.

- Choice of production strategy when export ships arrive late.

- Choice of production strategy when one or more wells unexpectedly shuts down. These are both important and plausible scenarios, and production strategies are chosen so as to minimize loss in profit compared to the nominal case. This is almost the same as fulfilling future contracts while minimizing the plant's operational costs like coolingand electricity use.

\subsection{Model components}

We assume that it is possible to describe plant performance in a meaningful way without explicitly including detailed pressure-dynamics. Hence, it is possible to solve the model sequentially starting with the wells, continuing with the multiphase pipeline, the slug catcher and ending with the storage tanks as depicted in Fig.1. This implies that the whole model can be solved very efficiently.

In the real plant there are several recycle flows. In the model this is avoided by operating with "perfect” splits and by aggregating cost and delays of these recycle flows into the bigger units. It is assumed that the aggregated plant model captures these plant dynamics with sufficient accuracy without including complex recycle flows and imperfect splits. As a side remark these dynamics are very important to ensure quality and safety under production, but do not influence the overall dynamics significantly.

All model components use static models, except the multiphase pipeline, the slug catcher and the storage tanks. The pipeline dynamics are significant in some cases since its settling time is about 10 hours. Instead of resorting to a complex multiphase flow model, the pipeline model is a simple $2^{\text {nd }}$ order system which accounts for the fact that liquid holdup varies with the flow rate and it also incorporates the main pipeline dynamics. 
The decision variables, or control inputs, in the complete model are:

- The gas condensate flow rate from each wells (9 control inputs).

- The amount of energy produced in the gas turbine (1 control input).

- The gas and condensate output flow rates from the slug catcher (2 control inputs).

- Export flow rates of LNG, LPG and condensate, respectively, to appropriate ships (3 control inputs).

The mass flow which is tracked by the model is composed of 8 different components. These components are $\mathrm{CO}_{2}, \mathrm{~N}_{2}$, methane, ethane, propane, butane, condensate $\left(\mathrm{C}_{5^{+}}\right)$and water. Some components have been neglected; our hypothesis being that it does not reduce the quality of our results. The complete model includes mass component flow rates in and out of each module, and energy consumption, if applicable, in each component. Some modules like the LNG and LPG cooling compressors consume a significant amount of electrical energy. Detailed information can be found in (Dahl 2007).

\subsection{Implementation}

The model is implemented using MatLAB ${ }^{\circledR}$. Each component has the same inputoutput structure given by

$$
\left\lfloor W_{\text {out } 1, i}, W_{\text {out } 2, i}, \ldots, W_{\text {outn }_{i}, i}, E_{i}\right\rfloor=\text { unit }_{i}\left(t, U_{i}, W_{\text {in }, i}\right)
$$

$W_{\text {out } 1, i}, E_{i}$ are the component-based output mass flow rate, and consumed electric and heat energy during a time period. $n_{i}, U_{i}, W_{i n, i}$ refer to the number of output streams, decision variables and the component-based input mass flow rate, respectively. $t$ defines the simulation time period.

\section{The optimization problem}

The optimization problem is formulated so as to minimize some cost function on a predefined future time horizon, typically 1-2 weeks. The time axis is discretized, typically with a step size of 3-6 hours. The decision variables may vary on the prediction horizon, thereby increasing the dimension of the decision space considerably. To elaborate, letting 15 decision variables, as defined in section 3.2, vary every 6 hours on a one week prediction horizon provides us with a 420 decision variables.

The mathematical optimization problem consists of three parts; an objective function, the model and constraints. The objective function provides a quantitative measure of the cost incurred by reducing production, compared to full capacity, as it will be assumed that there is no market constraint.

The model of the production chain described earlier connects the whole system, in particular it couples the decision variables and the variables that define the cost function. Finally, the mathematical optimization problem includes a number of constraints, mainly capacity constraints in the individual components along the production chain. Due to page constraints we refer to Dahl (2007) for a comprehensive mathematical description. 
The optimization problem we are left with is a nonlinear program which is solved using a SQP-like algorithm, see e.g. Nocedal and Wright (2006).

An alternative approach is to use a mixed integer optimization formulation combined with piecewise linear models as by Tomasgard et.al. 2007 and Midthun 2007. Common for both approaches is that models for the whole value chain are used, and that the formulation of models have to be somewhat adjusted to fit the optimization formulation.

\section{Test scenario and results}

To evaluate a model-based optimization scheme the following test scenario was defined. It is assumed that LNG ships arrive at the export quay every 120 hours. Ships for LPG and condensate products arrive less frequently. The idea is to evaluate the incurred cost of one delayed LNG ship as a function of the delay time. This can be illustrated by Fig.3 where the first LNG ship is scheduled for arrival at time equals 48 hours and a delay is hence related to this time instant. It is assumed that later LNG ships arrive on schedule. The prediction horizon was chosen to be 256 hours and the decision variables might change every 6 hours.
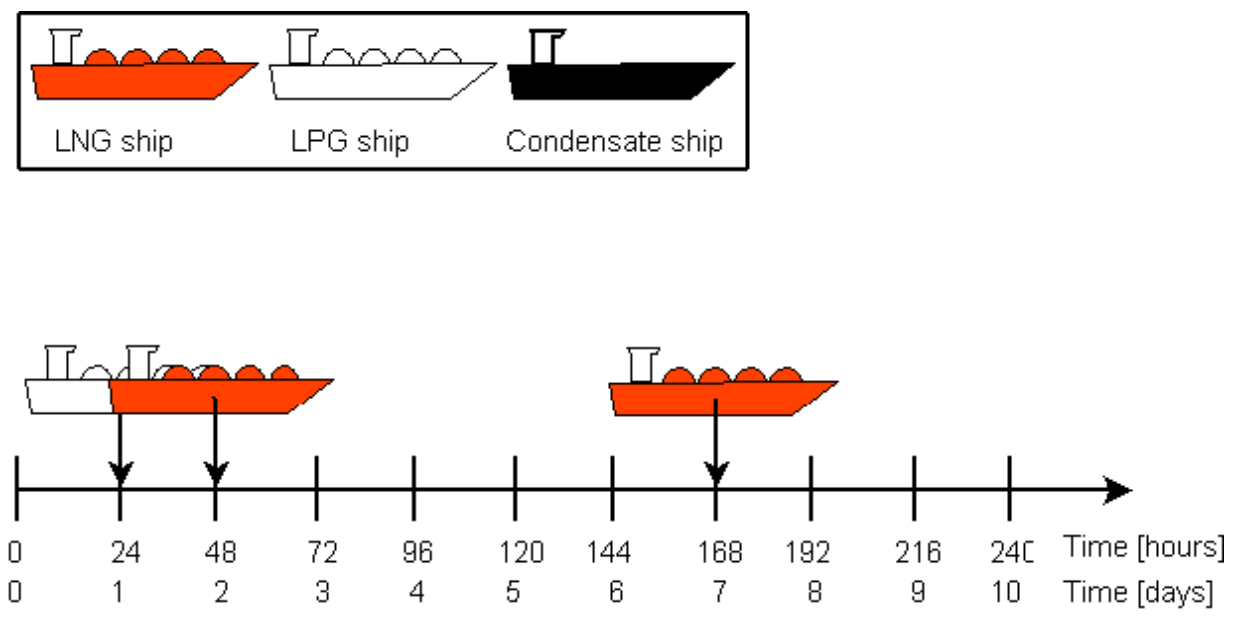

Figure 3. Normal ship arrivals

The production strategy computed by the optimizer is compared with a simple strategy where production is reduced such that the LNG storage is full when the LNG ship arrives and hence is ready to receive LNG.

Results are shown in Fig. 4 where the horizontal time axis corresponds to the time axis in Fig.3. These results show that no extra cost for short delays up until time equals 88 hours. The reason for this is that the LNG ships are scheduled to arrive when the storage 
tanks are about $74 \%$ full meaning that there is still spare capacity. For later arrivals, however, production must be reduced and sales income is lost.

The optimized production strategy provides a lower loss than the simple production strategy. The reason is that production is not only reduced, it is also redistributed between wells. The nine wells produce in pairs of three wells from three different reservoirs. Hence, the composition of the well streams differ. In particular, some wells produce more heavy components than others. Using this knowledge, which is embedded in the optimizer, the optimizer redistributes production such that LPG and condensate production is reduced less than LNG production as shown in Fig.5. This makes sense since there is no delay for the LPG and condensate ships in the presented scenario.

\section{Discussion of results}

This study shows that an approach using simple models connected according to the topology of the actual production system makes sense. An alternative approach would have been to use the high fidelity simulator of the Snøhvit system. This has not been studied herein. This would, however, probably give similar results to the ones shown above.

The results clearly show the importance of including the whole production chain from wells to export instead of the usual approach where the upstream part (the system from wells to the slug catcher) and the downstream part (from the slug catcher to export) are optimized individually. In the latter case the optimizer could not have anticipated the optimized production strategy as shown in Fig. 4 and 5 since this requires a model of the dependencies along the whole production chain.

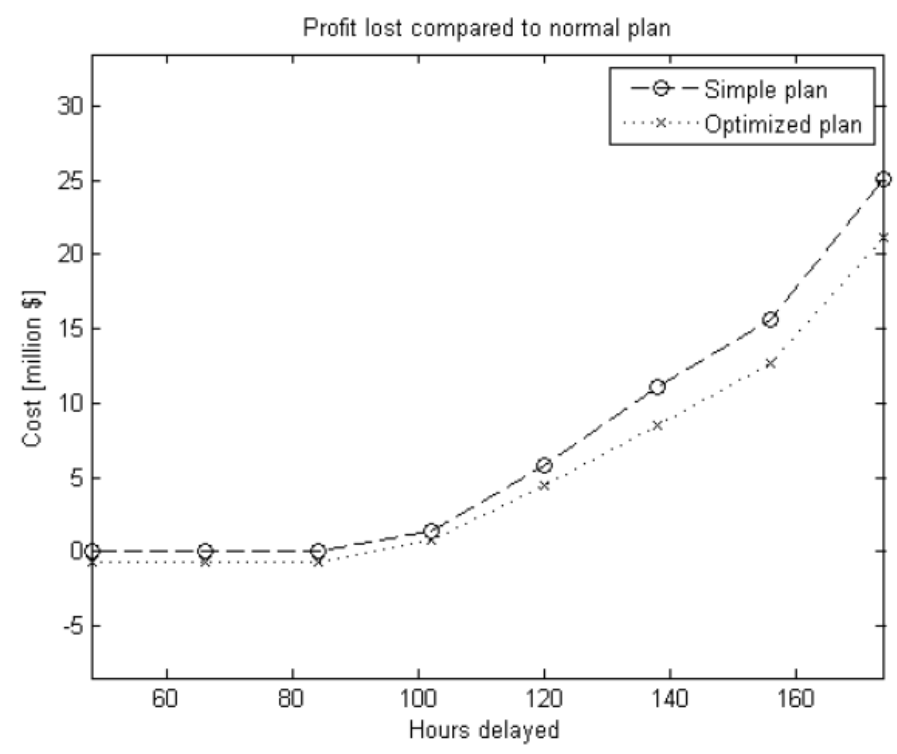

Figure 4. Cost incurred by a delayed LNG ship arrival. 


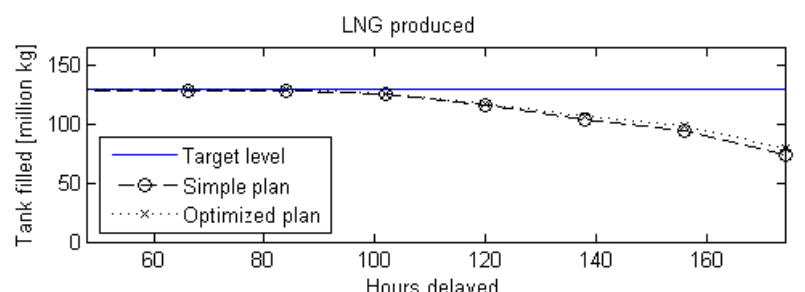

LPG produced
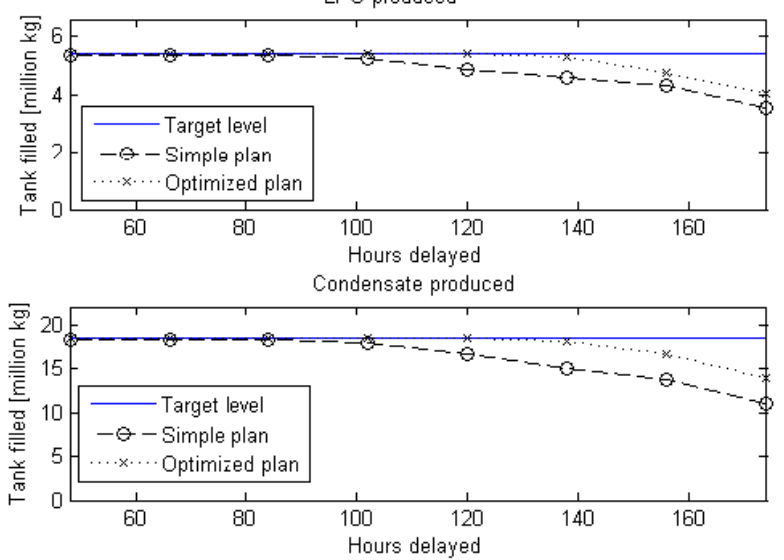

Figure 5. Production change due to delayed LNG ship arrival

One reason for stressing the use of simple models is the possible use of the optimizer in a model predictive control (MPC) strategy (see e.g. Qin and Badgewell, 1996). This implies re-optimizing the production strategy using a moving prediction horizon and gives a substantial increase in computational load. MPC introduces feedback control which may be important to account for uncertainties. An obvious uncertainty is the fact that the exact ship arrival time is not known. The arrival time may for instance be defined as a stochastic variable where candidate distributions might be the chi-squared or the F-distribution. In this case it might be necessary to formulate a stochastic optimization problem solving it as a multistage stochastic program (see e.g. Frauendorfer and Haarbrucker, 2002). This again increases computational load making it even more important to stick with a simple model.

The prediction horizon is quite long compared to the dynamics of the production chain model. Hence, the dynamic pipeline and slug-catcher models were replaced by static models. This reduced run time to $1 / 4$ of its original value without changing results significantly implying that this is a reasonable simplification. 


\section{Conclusion}

The simple model of the whole production chain combined with model-based optimization offers an interesting and feasible alternative to optimize production in the event of delayed ship arrival. Furthermore, the importance of bridging the gap between the upstream and the downstream domain by using a model of the whole value chain is shown.

\section{References}

T. Backx, O. Bosgra, W. Marquardt: "Integration of model predictive control and optimization of processes, IFAC Symposium “Advanced Control of Chemical Processes“, ADCHEM 2000, Pisa, Italy, 2000.

K. Dahl, “Optimization of the LNG-value chain”, Master thesis, Norwegian University of Science and Technology, 2007.

K. Frauendorfer, G. Haarbrucker: “Stochastic and global optimization”, Springer Verlag, 2002.

K.T. Midthun, ”Optimization models for liberalized natural gas markets”, PhD Thesis, Norwegian University of Science and Technology, NTNU, 2007:205.

J. Mula, R. Poler, J. P. García-Sabater, F. C. Lario: "Models for production planning under uncertainty: A review", International Journal of Production Economics, 2006.

J. Nocedal, S. J. Wright: “Numerical Optimization”, Springer Verlag, 2000.

B. Nygreen, M Christiansen, K. Haugen, T. Bjørkvoll \& Kristiansen, "Modeling Norwegian petroleum production and transportation”, Annals of Operations Research 82, 251-268, 1998.

S. J. Qin, T. A. Badgewell: “ An overview of industrial model predictive control technology”, CPC-V, Tahoe City, USA, 1996.

A. Tomasgard, F. Rømo, M. Fodstad, \& K. Midthun, “Optimization models for the natural gas value chain”, in G. Hasle, K.-A. Lie \& E. Quak, eds, ‘Geometric Modelling, Numerical Simulation, and Optimization: Applied Mathematics at SINTEF’, Springer, 2007. 